\title{
Serum concentrations of haptoglobin and haptoglobin-matrix metalloproteinase 9 (Hp-MMP 9) complexes of bovine calves in a bacterial respiratory challenge model
}

Christy J Hanthorn ${ }^{1}$, Grant A Dewell ${ }^{1 *}$, Renee D Dewell ${ }^{1,2}$, Vickie L Cooper ${ }^{1}$, Chong Wang ${ }^{1,4}$, Paul J Plummer ${ }^{1,3}$ and Jeffrey Lakritz ${ }^{5}$

\begin{abstract}
Background: Serum haptoglobin $(\mathrm{Hp})$ and haptoglobin matrix metalloproteinase 9 complexes (Hp-MMP 9) have been identified as biomarkers with diagnostic potential in cattle with conditions resulting in an acute inflammatory response. The purpose of this study was to evaluate potential diagnostic applications of serum Hp and Hp-MMP 9 concentrations in calves with BRD and establish a timeline for their detection in calves experimentally challenged with Bibersteinia trehalosi and Mannheimia haemolytica. Thirty-five cross bred dairy calves were inoculated via tracheal catheterization with either a PCR confirmed leukotoxin negative B. trehalosi isolate, a PCR confirmed leukotoxin positive B. trehalosi isolate, a Mannheimia haemolytica isolate, a combination of leukotoxin negative B. trehalosi and $M$. haemolytica, or a negative control. Serum samples were collected throughout the study. Calves were euthanized and necropsy performed on day 10 post inoculation.
\end{abstract}

Results: M. haemolytica inoculated calves had increased lung involvement. Serum Hp and Hp- MMP 9 concentrations were elevated compared to the other treatment groups. Increases in serum Hp and Hp-MMP 9 concentrations for the M. haemolytica group were significantly different from other study groups on day 7 of the study. B. trehalosi inoculated calves did not have increased lung involvement compared to control calves, but the leukotoxin positive $B$. trehalosi group demonstrated increased serum Hp-MMP 9 concentrations from day 3 to the end of the study compared to the pre-inoculation concentrations.

Conclusion: Serum Hp-MMP 9 concentration is a useful diagnostic tool for detecting early pulmonary inflammation in calves challenged with B. trehalosi and M. haemolytica. Serum Hp-MMP 9 may also be a useful tool in detecting subclinical pulmonary inflammation in challenged calves.

Keywords: Hp-MMP 9, Haptoglobin, MMP 9, Bovine Respiratory Disease, Pneumonia, Calf

\section{Background}

Bovine respiratory disease (BRD) has been shown to affect various biomarkers in cattle [1-7]. Two biomarkers that have been identified for diagnostic potential are serum haptoglobin (Hp), matrix metalloproteinase 9 (MMP 9), and the complexes that they are able to form (Hp-MMP 9) [1,6-9].

\footnotetext{
* Correspondence: gdewell@iastate.edu

'Department of Veterinary and Diagnostic Production Animal Medicine, College of Veterinary Medicine, lowa State University, Ames, IA 50011, USA Full list of author information is available at the end of the article
}

Free $\mathrm{Hp}$ is an alpha - 2 globulin that is primarily synthesized in the liver [3]. Free Hp's primary function is to bind to free hemoglobin in the blood. By scavenging the free hemoglobin, Hp helps to prevent oxidative tissue damage and conserve iron by returning the heme residue to the host's metabolic process. This scavenging mechanism also serves to decrease the risk of free hemoglobin being utilized for bacterial pathogen growth $[7,8]$.

MMP 9 is a collagenase belonging to the gelatinase B group [5]. The gelatinase B group is a family of MMP's 
that are zinc dependent proteinases capable of degrading at least one component of the extracellular matrix or basement membrane. This capability assists in the migration of white blood cells, mostly neutrophils, from the blood to the site of inflammation [4]. MMP 9 is stored in tertiary granules within the bovine neutrophils and is released when neutrophil degranulation is induced by either chemical or microbial stimuli $[1,5,10,11]$. After release, MMP 9 is able to cleave interleukin 8 , if present in the microenvironment into active interleukin 8 . This creates a positive feedback loop for neutrophil migration [10]. While only neutrophils have been demonstrated to store MMP 9 for immediate release [8], other cells such as alveolar macrophages can be induced to produce MMP 9 [5,12].

Free Hp and MMP 9 are secreted by multiple cellular sources in response to a variety of challenges; Hp-MMP 9 complexes have been shown to form exclusively in neutrophils [1]. These complexes are stored until neutrophil degranulation at which time they are released $[1,6,8]$. Hp has been demonstrated to have a high diagnostic sensitivity for detecting infectious or inflammatory conditions. Due to its poor specificity, it cannot be used as the primary diagnostic test for any one condition. Free $\mathrm{Hp}$ is also poor at differentiating between acute and chronic inflammation [1,7]. Free MMP 9 is more specific to acute inflammation, but it is not specific enough to differentiate between healthy and diseased cattle $[1,4]$. Hp-MMP 9 complexes are capable of differentiating between acute and chronic inflammation as their release from neutrophils is associated with acute inflammatory responses $[1,8]$.

Various biomarkers potentially associated with BRD have been studied. In previous studies $\mathrm{Hp}$ was demonstrated to increase after calves were exposed to Mannheimia haemolytica via intra-tracheal inoculation with the earliest detection occurring at 24 hours, and peak concentrations occurring at 3 days post inoculation [6,7]. In another challenge study, increases in serum Hp concentrations in calves infected with BVDV were not detected until 7-9 days post infection [7]. Serum concentrations of Hp-MMP 9 have not been evaluated in a BRD challenge study. The purpose of this study was two-fold: to evaluate the diagnostic applications of serum Hp-MMP 9 concentrations in calves with $\mathrm{BRD}$ and to establish a timeline for their detection in calves undergoing experimental challenge with Bibersteinia trehalosi and Mannheimia haemolytica.

\section{Methods}

Prior to initiating the study, the protocol was approved by the Iowa State University Institutional Animal Care and Use Committee (IACUC 8-11-7187-B) and the Institutional Biosafety Committee (IBC\#11-D-0017-A). This study is a hypothesis generating study representing a secondary use of calves enrolled in a challenge study designed to evaluate the pathogenicity of Bibersteinia trehalosi in respiratory disease among bovine calves [13]. The rationale for the secondary use of these calves to meet additional objectives is consistent with using the $3 \mathrm{R}$ principles to maximize information obtained from animal research [14].

Individually housed calves were inoculated via tracheal catheterization with of either a PCR (lktA) confirmed leukotoxin negative $B$. trehalosi isolate (8 calves), a PCR confirmed leukotoxin positive B. trehalosi isolate (8 calves), a Mannheimia haemolytica isolate (7 calves), a combination of leukotoxin negative B. trehalosi and M. haemolytica (8 calves), or a negative control (4 calves) as previously described [13]. The B. trehalosi isolates were field strains obtained from diagnostic submissions and the M. haemolytica isolate was a proprietary leukotoxin positive isolate. Calves were inoculated with $20 \mathrm{ml}$ of a Brain Heart Infusion broth containing approximately $2.5 \times 10^{9} \mathrm{CFU}$ of bacterial per milliliter.

Blood samples were collected from each calf via jugular venipuncture on Days 1 (pre-inoculation), 3, 5, 7, 9, and 10 (immediately prior to euthanasia). Blood samples were centrifuged at $4000 \mathrm{rpm}$ for 15 minutes. Following centrifugation, samples were placed on ice before being transferred, using a transfer pipette, to an appropriately labeled cryovial. Boxes containing cryovials were placed immediately in an ultra-low freezer and stored at $-70^{\circ} \mathrm{C}$ until being shipped to The Ohio State University on dry ice for analysis.

All surviving calves were euthanized on day 10 of the study, necropsied, and evaluated for percent abnormal lung involvement as previously described [13].

\section{Serum Haptoglobin-Matrix metalloproteinase 9 (Hp-MMP 9) ELISA assay}

Bovine Hp-MMP 9 complexes were determined as described previously $[1,6,15]$. All serum samples were diluted 1:5 with sample diluent (TBS $+1 \%$ Bovine serum albumin $+0.05 \%$ Tween 20). After blocking the wells ( $4^{\circ} \mathrm{C}$ for 120 minutes), known concentrations of $\mathrm{Hp}$ MMP 9 (serum, pre-characterized and shown to contain $912.6 \mathrm{ng} / \mathrm{mL}$ Hp-MMP 9) and the challenged calf serum samples were added to wells. Serum from healthy cows was used as a negative control.

\section{Serum total haptoglobin ELISA assay}

Serum Hp concentrations were determined as described (Bovine haptoglobin 96-well ELISA. Life Diagnostics, West Chester, PA 19380) using commercial Bovine haptoglobin ELISA test kits, according to manufacturer's instructions. Standard curves were prepared using purified bovine haptoglobin standard $(2.5 \mu \mathrm{g} / \mathrm{mL})$ included with the kit at a concentration range from $7.8-250 \mathrm{ng} / \mathrm{mL}$. 
Serum samples were diluted according to the kit instructions (1:2,000 dilution) and were run in duplicate. Controls included were normal bovine serum, 5\% BSA in TBS and blank wells. Linear regression of the Hp calibrator concentration versus absorbance was used to determine the equation for the line. The slope and intercept of this line was used to calculate the concentration of serum total $\mathrm{Hp}$ in the unknown animal samples. These concentrations were corrected for the dilution factor $(\times 2,000)$ and the concentrations reported in $\mu \mathrm{g} / \mathrm{mL}$.

\section{Statistical analysis}

Hp- MMP 9 and Hp were analyzed by repeated measures analysis of variance (ANOVA), with treatment, time, and their interaction as fixed effects and animal as subject of repeated measures. Lung involvement was analyzed by analysis of variance model, with treatment as explanatory variable. Correlations were calculated among the values of lung involvement, Hp, and Hp-MMP 9 for both $\mathrm{Hp}$ and Hp-MMP 9 serum concentrations as both ranked and absolute values prior to the challenge as well as averaged over the time of the study. SAS ${ }^{\bullet}$ Version 9.2 (SAS ${ }^{\bullet}$ Institute Inc., Cary, NC, USA) was used in analyses. A p-value $<0.05$ was considered significant.

\section{Results and discussion}

\section{Lung involvement}

Prior to inoculation all calves appeared clinically normal. Pasteurella multocida was isolated from pharyngeal swab samples from 34 of 35 calves. M. haemolytica was isolated from 8 of 35 calves. $M$. haemolytica positive pharyngeal swab calves were spread across all five treatment groups (1/4 in control group, $1 / 8$ in leukotoxin negative $B$. trehalosi group, $2 / 8$ in leukotoxin positive $B$. trehalosi group, $1 / 7$ in $M$. haemolytica group, 3/8 in leukotoxin negative $B$. trehalosi and $M$. haemolytica combination group).

Six of the 35 enrolled calves were euthanized prior to day 9 of the study. Three calves (3/7) from the $M$. haemolytica group (days 2, 3 and 3) and three calves (3/8) from the leukotoxin negative B. trehalosi and $M$. haemolytica combination treatment (days 3, 5 and 8) were euthanized according to protocol because of a clinical assessment of moribund.

The mean estimated percent lung involvement was highest for the $M$. haemolytica group (49\%). The mixed infection group of leukotoxin negative B. trehalosi and $M$. haemolytica had a mean lung involvement of $26 \%$. The leukotoxin positive $B$. trehalosi had a mean lung involvement of $18 \%$ while the leukotoxin negative $B$. trehalosi mean lung involvement was estimated to be $13 \%$. The control group had a mean lung involvement estimate of $13 \%$. There was evidence of a statistically significant $(\mathrm{p}=0.018)$ difference for mean percent total lung involvement between the $M$. haemolytica group and the leukotoxin negative $B$. trehalosi group. There were no significant differences between the other treatment groups. Even though the control group had a lower mean percent lung involvement than the leukotoxin negative $B$. trehalosi treatment group, the low number (4 calves) of study subjects in the control group did not have sufficient power to make it significantly different from the $M$. haemolytica treatment group. The mean and median values were similar in all treatment groups except the mixed infection group. This group had a high amount of variability within it as evidenced by a mean percent lung involvement of $26 \%$ and a median percent lung involvement of $5 \%$. The high variability made drawing conclusions difficult in this group (Figure 1).

\section{Free $\mathrm{Hp}$}

Other studies have found that serum $\mathrm{Hp}$ is nearly undetectable in healthy cattle [8]. Concentrations up to about $200 \mu \mathrm{g} / \mathrm{mL}$ are considered acceptable for healthy animals $[2,7,9,16]$. Concentrations between about 200 and $400 \mu \mathrm{g} / \mathrm{mL}$ are associated with mild inflammation and concentrations greater than about $400 \mu \mathrm{g} / \mathrm{mL}$ suggest severe inflammation [7]. Serum $\mathrm{Hp}$ concentration does not differ with age or sex in cattle $[2,7,9]$.

In this study, 11 calves (2/4 in the control group, 2/8 in the leukotoxin negative $B$. trehalosi group, $4 / 8$ in the leukotoxin positive $B$. trehalosi group, $1 / 7$ in the $M$. haemolytica group, and $2 / 8$ in the leukotoxin negative $B$. trehalosi and $M$. haemolytica combination group) had serum Hp concentrations greater than $200 \mu \mathrm{g} / \mathrm{mL}$ prior to inoculation. Two of the calves had low serum concentrations of Hp-MMP 9 indicating the presence of chronic inflammation. M. haemolytica was cultured from the pharyngeal swabs of both calves. Nine of the calves with high serum concentrations of $\mathrm{Hp}$ also had high serum concentrations of Hp-MMP 9 indicating the presence of chronic inflammation which was associated with an acute inflammatory event. Three of these calves were culture positive for $M$. haemolytica from pharyngeal swabs. Nineteen calves had no detectible serum Hp prior to inoculation.

The interaction between treatment group and bleeding date was a statistically significant effect $(\mathrm{p}=0.01)$. The mean serum $\mathrm{Hp}$ concentration for the leukotoxin positive $B$. trehalosi treatment group was significantly $(\mathrm{p}=0.008)$ different from the other treatment groups for the pre-inoculation bleeding date, indicating that more calves with evidence of chronic inflammation prior to commencement of the study were randomly assigned to this treatment group. On the fourth and sixth bleeding dates (days 7 and 10) there was a significant $(\mathrm{p}<0.05)$ difference in the mean Hp concentration between the $M$. haemolytica treatment group and the 


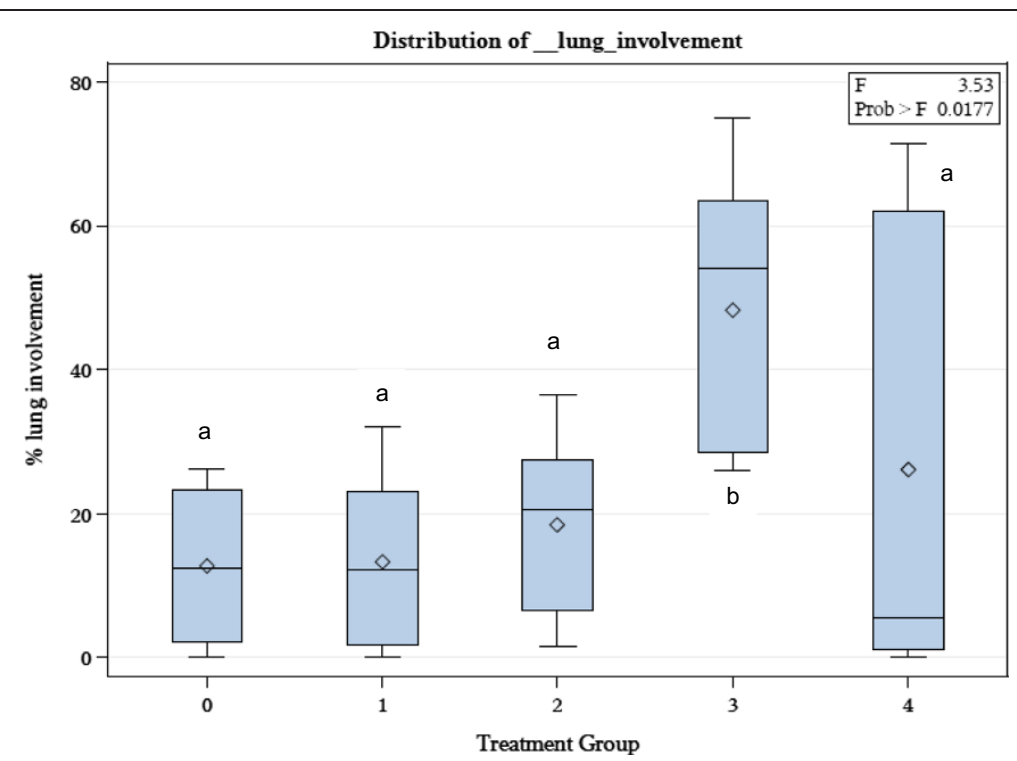

Figure 1 Distribution of lung involvement. Distribution of percent lung involvement by treatment group. Treatment group 0: Negative control group, Treatment group 1: leukotoxin negative B. trehalosi group, Treatment group 2: leukotoxin positive B. trehalosi group, Treatment group 3: M. haemolytica group, Treatment group 4: Combination of leukotoxin negative $B$. trehalosi and $M$. haemolytica group. Means with different letters are significantly different $(p<0.05)$. Diamonds represent the group mean. Boxes represent the middle two quartiles of individual values, divided by the center line which represents the median. Bars adjacent to the boxes represent the outer two quartiles of individual values.

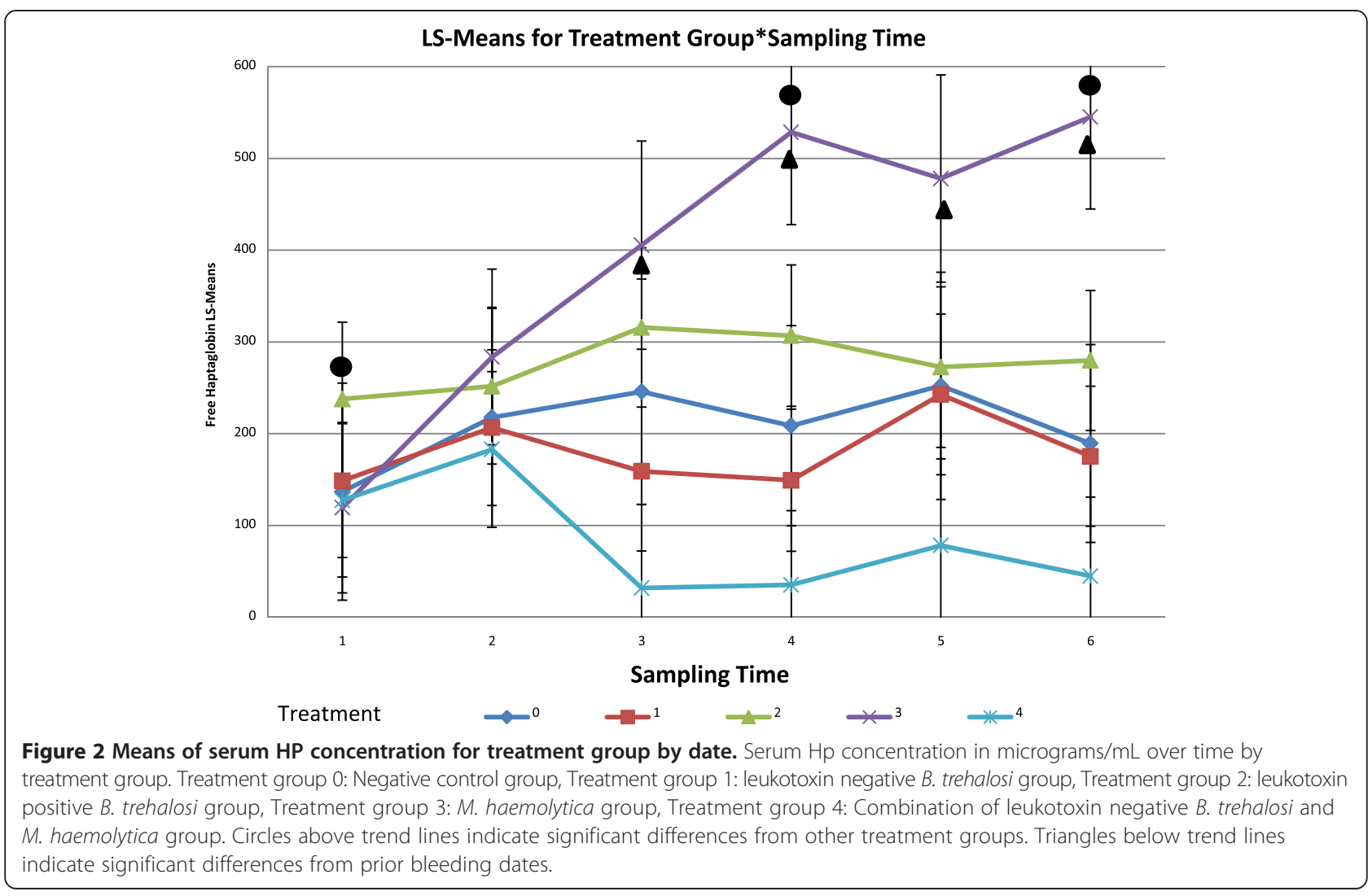


leukotoxin negative $B$. trehalosi treatment group and the leukotoxin negative $B$. trehalosi and M. haemolytica combination group. The $M$. haemolytica treatment group demonstrated significant elevations in serum $\mathrm{Hp}$ concentrations from the first two bleeding dates to the third bleeding date (day 5) $(\mathrm{p}=0.02)$. The elevation in serum Hp concentration remained significant $(\mathrm{p}<0.02)$ throughout the study. This finding is in agreement with other studies that have demonstrated an increase in serum Hp after $M$. haemolytica infection $[4,6]$. Concentrations in this study peaked on day 7 rather than day 3 as previously reported. A decrease in the serum $\mathrm{Hp}$ concentration for the mixed infection group can be observed between the second and third bleeding dates (days 3 and 5). This observation supports findings by Dassanayake et al. suggesting that B. trehalosi is capable of inhibiting the growth of M. haemolytica in vivo [17]. In this study the difference in serum Hp concentration is not significantly different from the other treatment groups. Two calves from this treatment group were euthanized before the third bleeding time point. The smaller treatment group likely did not have enough power for a statistical difference to be observed (Figure 2).

\section{Hp-MMP 9}

The mean serum Hp-MMP 9 concentration for all treatment groups prior to inoculation was $104 \mathrm{ng} / \mathrm{mL}$, with a minimum value of $0 \mathrm{ng} / \mathrm{mL}$, a maximum value of $752.2 \mathrm{ng} / \mathrm{mL}$, and a median value of $4.15 \mathrm{ng} / \mathrm{mL}$. Little to no Hp-MMP 9 should be detectible in the serum of healthy calves [6]. Sixteen calves had no detectible serum Hp-MMP 9 concentration prior to inoculation. For this study a serum concentration greater than $20 \mathrm{ng} / \mathrm{mL}$ was considered high. Thirteen calves had high serum HpMMP 9 concentrations prior to inoculation. Four of the thirteen calves had low serum concentrations of $\mathrm{Hp}$, suggesting the presence of a mild acute inflammatory process. None of these 4 calves were culture positive for $M$. haemolytica from the pharyngeal swabs. Nine of the thirteen calves had high serum concentrations of $\mathrm{Hp}$ and were discussed previously.

Treatment group, bleeding date, and their interaction were all significant $(\mathrm{p}<0.01)$ effects. On the fourth bleeding date (day 7) the mean serum Hp-MMP 9 concentration for the $M$. haemolytica group became significantly different from the negative control, the leukotoxin negative $B$. trehalosi, and the leukotoxin negative $B$. trehalosi and $M$. haemolytica combination groups $(\mathrm{p}=0.01, \mathrm{p}=0.009$, and $\mathrm{p}=0.004$ respectively). This difference continued throughout the study. On the fifth bleeding date (day 9) the mean serum Hp-MMP 9 concentration for the $M$. haemolytica group became significantly different from the leukotoxin positive $B$. trehalosi group $(\mathrm{p}=0.006)$ and remained different throughout the rest of the study. The M. haemolytica group demonstrated significant elevations in serum Hp-MMP 9 concentrations from the first three bleeding dates to the last three bleeding dates $(\mathrm{p}<0.02)$. The leukotoxin positive $B$. trehalosi treatment group demonstrated a small but significant $(\mathrm{p}<0.004)$ elevation in serum HpMMP 9 concentrations from the pre-inoculation bleeding date to the second bleeding date (day 3) that continued throughout the study (Figure 3). This finding is consistent with other studies that have demonstrated the rapid recruitment and accumulation of neutrophils at the onset of BRD [6] as well as the action of leukotoxins to stimulate the active degranulation of bovine neutrophils [5].

\section{Correlations}

There was statistically significant $(\mathrm{p}<0.0001)$ evidence of a strong $(r=0.7)$ correlation between $\mathrm{Hp}$ and Hp-MMP 9 serum concentrations as both ranked and absolute values prior to inoculation. Evidence of a relationship remained statistically significant over time $(\mathrm{p}<0.0001)$, but the strength of the relationship varied depending on if a Pearson (absolute values) or Spearman (ranked data) correlation coefficient was calculated. The Pearson correlation coefficient was $r=0.63$; whereas, the Spearman correlation coefficient was $r=0.79$. This strong relationship was expected since both substances have been established as biomarkers for inflammation and the test for free $\mathrm{Hp}$ measures Hp-MMP 9 contribution also.

There was statistically significant evidence $(\mathrm{p}<0.05)$ of a moderate correlation $(r=0.44$ and $r=0.35)$ that became stronger over time $(r=0.67$ and $r=0.48)$ between Hp-MMP 9 and percent lung involvement for both ranked and absolute values respectively. There was statistically significant evidence $(\mathrm{p}=0.04)$ of a moderate $(\mathrm{r}=0.35)$ correlation between free $\mathrm{Hp}$ and percent lung involvement for ranked, but not absolute values prior to inoculation. This correlation became stronger $(\mathrm{r}=0.54, \mathrm{p}=0.0008)$ when values were averaged over the time of the study. The correlation between free $\mathrm{Hp}$ and percent lung involvement for absolute data was also statistically significant when averaged over time $(\mathrm{r}=0.41, \mathrm{p}=0.015)$.

This correlation data indicates that Hp-MMP 9 serum concentrations may be a good ante-mortem diagnostic indicator of lung damage that is found at the time of the calf's death. This data also supports the conclusion from other studies that Hp-MMP 9 serum concentration is a better diagnostic test for lung damage than free Hp serum concentration [1]. Future studies should be conducted to determine reference ranges of serum Hp-MMP 9 for different levels of disease severity.

\section{Conclusion}

All study calves appeared to be clinically normal prior to inoculation in this study. Culture results of pharyngeal 


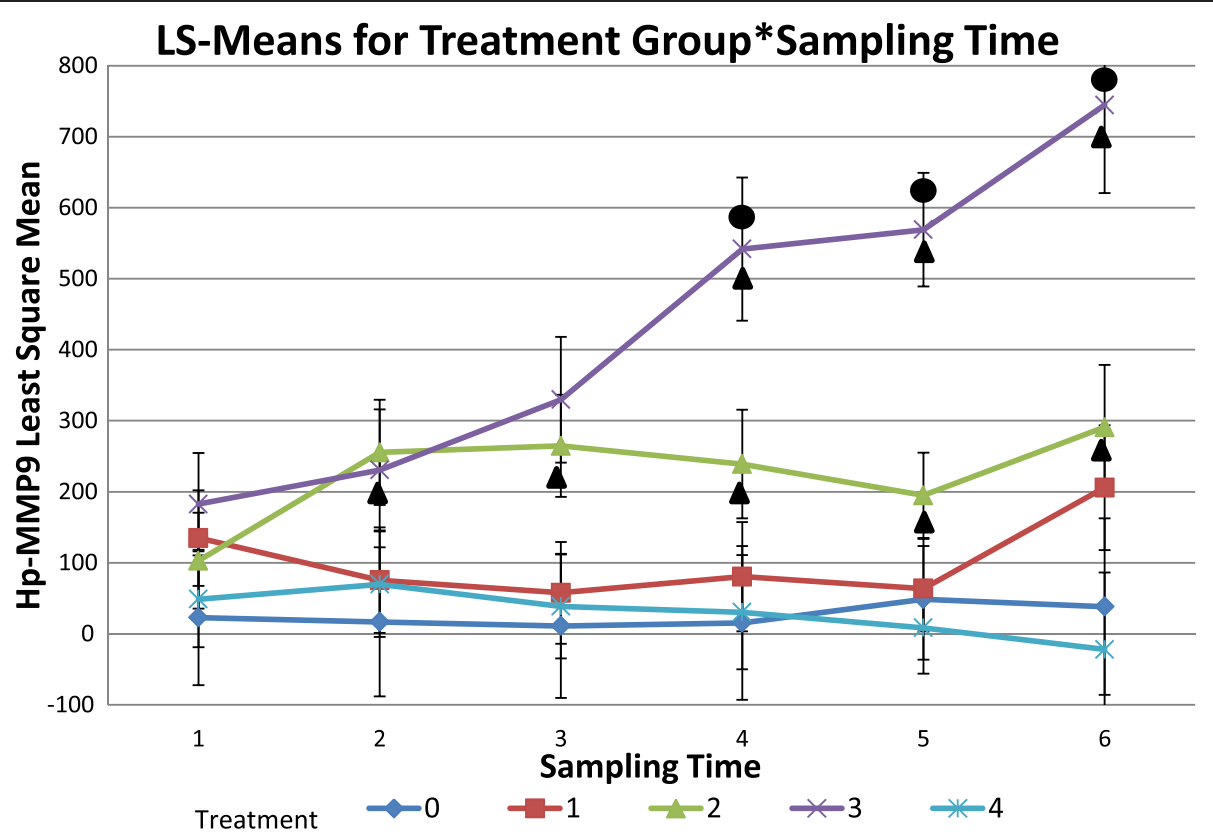

Figure 3 Means of serum Hp-MMP 9 concentration for treatment group by date. Serum Hp-MMP 9 concentration in nanograms $/ \mathrm{mL}$ over time by treatment group. Treatment group 0: Negative control group, Treatment group 1: leukotoxin negative B. trehalosi group, Treatment group 2: leukotoxin positive B. trehalosi group, Treatment group 3: M. haemolytica group, Treatment group 4: Combination of leukotoxin negative B. trehalosi and $M$. haemolytica group. Circles above trend lines indicate significant differences from other treatment groups. Triangles below trend lines indicate significant differences from prior bleeding dates.

swabs taken from calves prior to inoculation showed that all but one calf was positive for P. multocida. The presence of this bacterium did not appear to have an impact on pre-inoculation values of serum $\mathrm{Hp}$ or $\mathrm{Hp}$ MMP 9 since many of the calves had serum concentrations of these two biomarkers that were within limits considered acceptable for healthy animals $[2,7,9,16]$. The pharyngeal swabs for 8 calves were culture positive for $M$. haemolytica prior to inoculation. This finding could have potentially affected the development of lung lesions as well as increases in serum $\mathrm{Hp}$ and Hp-MMP 9 concentrations in calves that were members of treatment groups other than the $M$. haemolytica group. The effect was not large enough to preclude seeing statistically significant results from the challenge in the $M$. haemolytica group. The development of a rapid, inexpensive test for serum Hp-MMP 9 concentrations could have clinical applications as a screening test for subject enrollment in BRD studies. Subjects with elevated serum concentrations could be excluded from participation.

Significant differences were observed between the $M$. haemolytica group and the other treatment groups in percent total lung involvement, serum $\mathrm{Hp}$, and serum Hp-MMP 9 concentrations. The difference in percent total lung involvement demonstrates that the inoculation technique was appropriate for this challenge model. The increase in serum Hp and Hp-MMP 9 concentrations at day 7 are consistent with other reports of these two biomarkers having diagnostic potential with respect to $B R D$. Results suggest a different, slightly later, timeframe for using them as diagnostic tools.

No significant difference was observed between the leukotoxin positive $B$. trehalosi treatment group and the negative control, leukotoxin negative $B$. trehalosi, and combination of leukotoxin negative $B$. trehalosi and $M$. haemolytica groups with respect to percentage of total lung involvement or serum $\mathrm{Hp}$ concentrations. A small but significant increase was noted in the serum HpMMP 9 concentrations of the leukotoxin positive $B$. trehalosi treatment group from day 1 to day 3 , indicating that serum Hp-MMP 9 concentrations may be a more sensitive test for pulmonary inflammation than visible lung damage or serum $\mathrm{Hp}$ concentrations. This finding also provides evidence that the leukotoxin positive strain of $B$. trehalosi induced more neutrophil degranulation leading to a higher release of Hp-MMP 9 complexes than the leukotoxin negative strain of $B$. trehalosi. In this study the earlier rise in the serum Hp-MMP 9 concentration for the leukotoxin positive B. trehalosi treatment group than for the $M$. haemolytica treatment group may indicate that bovine neutrophils respond faster to a $B$. trehalosi infection than to a M. haemolytica infection.

The stronger significant correlation between serum Hp-MMP 9 concentration and percent lung involvement than between serum Hp concentration and percent lung involvement support the conclusions of other studies 
that serum Hp-MMP 9 concentrations can be an effective tool for early diagnosis of BRD [6] and that it is a better diagnostic tool for acute inflammation than serum Hp [1]. Future studies to determine a reference range for serum Hp-MMP 9 concentration and to develop a rapid, inexpensive test would help to make serum Hp-MMP 9 concentration a clinically useful diagnostic tool.

\section{Abbreviations}

Hp-MMP 9: Haptoglobin-matrix metalloproteinase 9; Hp: Haptoglobin; MMP 9: Matrix metalloproteinase 9; BRD: Bovine respiratory disease; BVDV: Bovine viral diarrhea virus; PI: Persistently infected; PCR: Polymerase chain reaction; M. haemolytica: Mannheimia haemolytica; $B$. trehalosi: Bibersteinia trehalosi; P. multocida: Pasteurella multocida.

\section{Competing interests}

The authors declare that they have no competing interests.

\section{Authors' contributions}

GD and PP conceived and coordinated the experiment. $\mathrm{CH}$ and RD performed the experiment. VC evaluated lungs. CW conducted statistical analysis. JL oversaw the ELISA procedures. All authors read and approved the final manuscript.

\section{Acknowledgements}

The authors thank Boehringer Ingelheim Vetmedica, Inc. for their support of research through the lowa State University Foundation. The authors also thank the Academy of Veterinary Consultants for their financial support through the Armstrong Memorial Scholarship. Jessica Ninneman is acknowledged for her excellent laboratory assistance.

\section{Author details}

'Department of Veterinary and Diagnostic Production Animal Medicine, College of Veterinary Medicine, lowa State University, Ames, IA 50011, USA. ${ }^{2}$ Department of Veterinary Microbiology and Preventive Medicine, Center for Food Security and Public Health, College of Veterinary Medicine, lowa State University, Ames, IA 50011, USA. ${ }^{3}$ Department of Veterinary Microbiology and Preventive Medicine, College of Veterinary Medicine, lowa State University, Ames, IA 50011, USA. Department of Statistics, College of Liberal Arts and Sciences, lowa State University, Ames, IA 50011, USA. ${ }^{5}$ Department of Veterinary Clinical Sciences, College of Veterinary Medicine, The Ohio State University, Columbus, $\mathrm{OH}$ 43210, USA.

Received: 11 July 2014 Accepted: 19 November 2014 Published online: 06 December 2014

\section{References}

1. Bannikov GA, Hinds CA, Rajala-Schultz PJ, Premanandan C, Rings DM, Lakritz J: Serum haptoglobin-matrix metalloproteinase 9 (Hp-MMP 9) complex as a biomarker of systemic inflammation in cattle. Vet Immunol Immunopathol 2011, 139(1):41-49

2. Godson DL, Campos M, Attah-Poku SK, Redmond MJ, Cordeiro DM, Seth MS, Harland RJ, Babiuk LA: Serum haptoglobin as an indicator of the acute phase response in bovine respiratory disease. Vet Immunol Immunopathol 1996, 51(3/4):277-292.

3. Nazifi S, Rezakhani A, Koohimoghadam M, Ansari-Lari M, Esmailnezhad Z Evaluation of serum haptoglobin in clinically healthy cattle and cattle with inflammatory diseases in Shiraz, a tropical area in Southern Iran. Bulgarian J Vet Med 2008, 11(2):95-101.

4. Simonen-Jokinen TLM, Eskelinen UM, Hartel HM, Nikunen SK, Saloniemi HS, Maisi PS: Gelatinolytic matrix metalloproteinases-2 and -9 in tracheobronchial lavage fluid obtained from calves with concurrent infections of Pasteurella multocida and Mycoplasma bovirhinis. Am J Vet Res 2005, 66(12):2101-2106.

5. Starr AE, Dan T, Minhas K, Shewen PE, Coomber BL: Potential involvement of gelatinases and their inhibitors in Mannheimia haemolytica pneumonia in cattle. Infect Immun 2004, 72(8):4393-4400.

6. Theurer ME, Anderson DE, White BJ, Miesner MD, Mosier DA, Coetzee JF, Lakritz J, Amrine DE: Effect of Mannheimia haemolytica pneumonia on behavior and physiologic responses of calves during high ambient environmental temperatures. J Anim Sci 2013, 91(8):3917-3929.

7. Tothova C, Nagy O, Kovac G: The Use of Acute Phase Proteins as Biomarkers of Diseases in Cattle and Swine. In Acute Phase Proteins. Edited by Janciauskiene S. Rijeka Croatia: InTech; 2013:103-138.

8. Bannikov GA, Mattoon JS, Abrahamsen EJ, Premanandan C, Green-Church $\mathrm{KB}$, Marsh AE, Lakritz J: Biochemical and enzymatic characterization of purified covalent complexes of matrix metalloproteinase- 9 and haptoglobin released by bovine granulocytes in vitro. Am J Vet Res 2007, 68(9):995-1004

9. Seppa-Lassila L, Orro T, LePage JP, Soveri T: Reference values for acute phase proteins in calves and their clinical application. Vet Rec 2013, 173(13):319.

10. Sporer KRB, Burton JL, Earley B, Crowe MA: Transportation stress in young bulls alters expression of neutrophil genes important for the regulation of apoptosis, tissue remodeling, margination, and anti-bacterial function. Vet Immunol Immunopathol 2007, 118(1/2):19-29.

11. Li X, Zhao X, Ma S: Secretion of $92 \mathrm{kDa}$ gelatinase (MMP-9) by bovine neutrophils. Vet Immunol Immunopathol 1999, 67(3):247-258.

12. Lakritz J, Marsh AE, Cockrell M, Smith MF, Tyler JW: Characterization of gelatinases in bronchoalveolar lavage fluid and gelatinases produced by alveolar macrophages isolated from healthy calves. Am J Vet Res 2004 65(2):163-172

13. Hanthorn CJ, Dewell RD, Cooper VL, Frana TS, Plummer PJ, Wang C, Dewell GA: Randomized Clinical Trial to Evaluate the Pathogenicity of Bibersteinia trehalosi in Respiratory Disease among Calves. In: BMC Veterinary Research. vol. 10. http://www.biomedcentral.com/1746-6148/10/ 89: BioMed Central; 2014.

14. Russell WMS, Burch RL: The principles of humane experimental technique. London UK: Methuen; 1959. xiv-238.

15. Van Engen NK, Stock ML, Engelken T, Vann RC, Wulf LW, Karriker LA, Busby WD, Lakritz J, Carpenter AJ, Bradford BJ, Hsu WH, Wang C, Coetzee JF: Impact of oral meloxicam on circulating physiological biomarkers of stress and inflammation in beef steers after long-distance transportation. J Anim Sci 2014, 92(2):498-510.

16. Chan P, Chu C, Fung H, Chuang S, Lin Y, Chu M, Lee S: Serum Haptoglobin concentration in cattle. J Vet Med Sci 2004, 66(1):43-46.

17. Dassanayake RP, Shanthalingam S, Subramaniam R, Herndon CN, Bavananthasivam J, Haldorson GJ, Foreyt WJ, Evermann JF, Herrmann-Hoesing LM, Knowles DP, Srikumaran S: Role of Bibersteinia trehalosi, respiratory syncytial virus, and parainfluenza-3 virus in bighorn sheep pneumonia. Vet Microbio/ 2013, 162(1):166-172.

\section{doi:10.1186/s12917-014-0285-5}

Cite this article as: Hanthorn et al:: Serum concentrations of haptoglobin and haptoglobin-matrix metalloproteinase 9 (Hp-MMP 9) complexes of bovine calves in a bacterial respiratory challenge model. BMC Veterinary Research 2014 10:285.

\section{Submit your next manuscript to BioMed Central and take full advantage of:}

- Convenient online submission

- Thorough peer review

- No space constraints or color figure charges

- Immediate publication on acceptance

- Inclusion in PubMed, CAS, Scopus and Google Scholar

- Research which is freely available for redistribution 